\title{
Cardiothoracic
}

Transplantation

\section{Early outcomes in human lung transplantation with Thymoglobulin or Campath-1H for recipient pretreatment followed by posttransplant tacrolimus near-monotherapy}

Kenneth R. McCurry, MD, ${ }^{a}$ Aldo lacono, MD, ${ }^{b}$ Adrianna Zeevi, PhD, ${ }^{\mathrm{a}, \mathrm{b}, \mathrm{c}, \mathrm{d}}$ Samuel Yousem, MD, ${ }^{\mathrm{c}}$ Alin Girnita, MD, Shahid Husain, MD, ${ }^{\mathrm{b}}$ Diana Zaldonis, BSN, ${ }^{\mathrm{a}}$ Bruce Johnson, MD, ${ }^{\mathrm{b}}$ Brack G. Hattler, MD, ${ }^{\mathrm{a}}$ and

Thomas E. Starzl, MD, PhD ${ }^{\mathrm{a}, \mathrm{d}}$

From the Departments of Surgery, ${ }^{\text {a }}$ Medicine, ${ }^{\mathrm{b}}$ and Pathology ${ }^{\mathrm{c}}$ and the Thomas E. Starzl Transplantation Institute, ${ }^{\mathrm{d}}$ The University of Pittsburgh Medical Center, Pittsburgh, Pa.

Read at the Eighty-fourth Annual Meeting of The American Association for Thoracic Surgery, Toronto, Ontario, Canada, April 25-28, 2004.

Received for publication April 20, 2004; revisions received Sept 8, 2004; accepted for publication Sept 30, 2004.

Address for reprints: Kenneth R. McCurry, MD, 200 Lothrop St, Suite C-700, Department of Surgery, University of Pittsburgh, Pittsburgh, PA 15213 (E-mail: mccurrykr@ upmc.edu).

J Thorac Cardiovasc Surg 2005;130:528-37

$0022-5223 / \$ 30.00$

Copyright (c) 2005 by The American Association for Thoracic Surgery

doi:10.1016/j.jtcvs.2004.09.040
Objectives: Acute and chronic rejection remain unresolved problems after lung transplantation, despite heavy multidrug immunosuppression. In turn, the strong immunosuppression has been responsible for mortality and pervasive morbidity. It also has been postulated to interdict potential mechanisms of alloengraftment.

Methods: In 48 lung recipients we applied 2 therapeutic principles: (1) recipient pretreatment with antilymphoid antibody preparations (Thymoglobulin [SangStat, Fremont, Calif] or Campath [alemtuzumab; manufactured by ILEX Pharmaceuticals, LP, San Antonio, Tex; distributed by Berlex Laboratories, Richmond, Calif]) and (2) minimal posttransplant immunosuppression with tacrolimus monotherapy or near-monotherapy. Our principal analysis was of the events during the critical first 6 posttransplant months of highest immunologic and infectious disease risk. Results were compared with those of 28 historical lung recipients treated with daclizumab induction and triple immunosuppression (tacrolimus-prednisone-azathioprine).

Results: Recipient pretreatment with both antilymphoid preparations allowed the use of postoperative tacrolimus monotherapy with prevention or control of acute rejection. Freedom from rejection was significantly greater with Campath than with Thymoglobulin $(P=.03)$ or daclizumab $(P=.05)$. After lymphoid depletion with Thymoglobulin or Campath, patient and graft survival at 6 months was $90 \%$ or greater. Patient and graft survival after 9 to 24 months is $84.2 \%$ in the Thymoglobulin cohort, and after 10 to 12 months, it is $90 \%$ in the Campath cohort. There has been a subjective improvement in quality of life relative to our historical experience.

Conclusion: Our results suggest that improvements in lung transplantation can be accomplished by altering the timing, dosage, and approach to immunosuppression in ways that might allow natural mechanisms of alloengraftment and diminish the magnitude of required maintenance immunosuppression.

$\mathrm{T}$ raditional immunosuppressive strategies for organ transplantation have involved, from the time of operation, the use of potent multidrug regimens, including a calcineurin inhibitor, high doses of prednisone, and an antimetabolite with or without a short course of antilymphoid antibody (induction). Despite the strong prophylactic immunosuppression, the incidence of acute rejection in the 
first 6 to 12 months after lung transplantation has remained high. ${ }^{1}$ Moreover, complications from the chronic immune depression and from drug-specific toxicities have been the rule rather than the exception, with infection-related mortality the most common cause of death in the first 3 years after transplantation. ${ }^{2}$ As a result, the 1- and 5-year patient survivals reported from national and international lung transplantation registries are $73 \%$ to $77 \%$ and $42 \%$ to $45 \%$, respectively. ${ }^{2,3}$

It has also been suggested that excessive early postoperative immunosuppression can potentially subvert postulated mechanisms of alloengraftment. ${ }^{4}$ Two therapeutic principles were applied in 48 lung recipients to avoid this selfdefeating consequence of treatment, beginning in June 2002. First, the lung recipients were infused with a single large dose of a potent antilymphoid preparation during the few hours preceding the operation or intraoperatively but before allograft reperfusion. The pretreatment was either with a rabbit antithymocyte globulin (Thymoglobulin; SangStat, Fremont, Calif; $n=38$ ) or with the broadly reacting humanized anti-CD52 monoclonal antibody alemtuzumab (Campath-1H [alemtuzumab], hereafter referred to as Campath; manufactured by ILEX Pharmaceuticals, LP, San Antonio, Tex; distributed by Berlex Laboratories, Richmond, Calif; $\mathrm{n}=10$ ). Second, the recipients were treated after transplantation with tacrolimus monotherapy alone or in combination with very low doses of prednisone (usually $\leq 5 \mathrm{mg} / \mathrm{d}$ ).

\section{Methods}

\section{Recipient and Donor Demographics}

All adult patients undergoing single- or double-lung transplantation or heart-lung transplantation at the University of Pittsburgh between June 2002 and September 2003 were managed with the protocol described below, except for 5 recipients in whom there were logistical difficulties. From June 2002 through June 2003, 37 recipients were pretreated with 4 to $7 \mathrm{mg} / \mathrm{kg}$ intravenous Thymoglobulin; a 38th patient was added in September 2003. Between June 2003 and August 2003, the second cohort of 10 patients was pretreated with $30 \mathrm{mg}$ of intravenous Campath instead of Thymoglobulin. Results are compared with those of 28 unselected patients who underwent single- or double-lung transplantation or heart-lung transplantation at the University of Pittsburgh between December 2001 and June 2002 who were managed with daclizumab (Zenapax) induction, followed by triple-drug immunosuppressive therapy.

The characteristics of the patients and donors in the 3 groups are shown in Table 1. There was no significant difference between groups for any recipient or donor variable. The indications for transplantation were broader in the Thymoglobulin-treated and daclizumab-treated cohorts than in the Campath group (Table 1). In the Thymoglobulin-treated cohort, other risk factors included 1 (3\%) patient with a preexisting, donor-specific, anti-class II antibody; 3 (8\%) patients with scleroderma; 1 (3\%) patient with cystic fibrosis with Burkholderia gladioli colonization; 2 (5\%) patients with sarcoidosis (odds ratio for death at 1 year of 2.03 by International Society for Heart and Ling Transplantation [ISHLT] Registry $^{2}$ ); and one recipient of a simultaneous liver.

\section{Immunosuppressive Protocol}

The antibody infusions (Thymoglobulin, Campath, or daclizumab) were initiated as soon as possible after confirmation of the acceptability of the donor organs. Thymoglobulin was begun slowly, with rate escalation every 30 minutes. Campath was infused at a steady rate over 2 hours. Patients who received Thymoglobulin or Campath were coadministered $1 \mathrm{~g}$ of methylprednisolone to suppress cytokine reactions. In all patients $250 \mathrm{mg}$ of methylprednisolone was administered immediately before lung allograft reperfusion ( 1 dose for single-lung or heart-lung recipients and 2 doses for double-lung recipients).

Thymoglobulin-treated and Campath-treated patients were given twice-daily oral tacrolimus (Prograf; Fujisawa Healthcare, Inc, Deerfield, Ill) beginning on postoperative day 1. Tacrolimus doses were adjusted to achieve a 12-hour trough level of 12 to $15 \mathrm{ng} / \mathrm{mL}$. All Thymoglobulin-treated patients received $5 \mathrm{mg} / \mathrm{d}$ prednisone beginning on postoperative day 1 . Five of the 10 Campath-treated patients were given $5 \mathrm{mg} / \mathrm{d}(\mathrm{n}=4)$ or $7.5 \mathrm{mg} / \mathrm{d}$ $(n=1)$ prednisone from postoperative day 1 because they were receiving corticosteroids preoperatively. The other 5 were treated with tacrolimus only. No patient received an antimetabolite.

For patients who were given daclizumab, the protocol called for 5 doses $(1 \mathrm{mg} / \mathrm{kg}$ each on day 0 , posttransplant day 7 , and posttransplant weeks 2, 4, and 6). These patients also received a methylprednisolone taper beginning the day of transplantation and extending over 5 days from $240 \mathrm{mg} / \mathrm{d}$ to $40 \mathrm{mg} / \mathrm{d}$, followed by 20 $\mathrm{mg}$ of prednisone daily. Twice-daily tacrolimus (target 12-hour trough level of $15-20 \mathrm{ng} / \mathrm{mL}$ ) and azathioprine $(2-3 \mathrm{mg} / \mathrm{kg}$ ) were begun the day of transplantation.

\section{Diagnosis and Treatment of Rejection}

Acute rejection was diagnosed by means of histologic examination of transbronchial or open lung biopsy specimens according to the revised working formulation for the histologic classification of pulmonary allograft rejection. ${ }^{5}$ The specimens were examined and graded by clinical pathologists according to our standard routine. Subsequent review of biopsy specimens was carried out at weekly conferences. Rejection was treated with corticosteroids (ie, 10-day oral prednisone taper [100 $\mathrm{mg}$ to previous baseline] or 1-2 doses of 500-1000 mg of methylprednisolone). In the Thymoglobulin- and Campath-conditioned patients, grade 2 acute rejection often was not treated in the absence of deterioration of allograft dysfunction unless there were histopathologic findings of issue injury. In contrast, all grade 2 acute rejection episodes were treated in the daclizumab cohort.

Higher-grade rejections were treated with 2 to 3 doses of $1000 \mathrm{mg}$ of methylprednisolone, with occasional augmentation of maintenance steroids or the addition of other baseline agents. After Campath became available in the autumn of 2002, it was used to treat steroid-resistant rejection. A few patients were switched from tacrolimus to cyclosporine because of drugspecific toxicity. 
TABLE 1. Demographics of lung transplant recipients in the Thymoglobulin, Campath, and daclizumab groups

\begin{tabular}{|c|c|c|c|c|}
\hline & Thymoglobulin & Campath & Daclizumab & $P$ value \\
\hline Number & 38 & 10 & 28 & \\
\hline Average age (y) & $47 \pm 13(25-68)$ & $55 \pm 11(37-70)$ & $49 \pm 14(22-65)$ & NS \\
\hline $\operatorname{Sex}(M / F)$ & $17 / 21$ & $7 / 3$ & $15 / 13$ & NS \\
\hline Underlying disease & & & & NS \\
\hline COPD & $12(37 \%)$ & $2(20 \%)$ & $8(29 \%)$ & \\
\hline CF & $8(21 \%)$ & & $5(18 \%)$ & \\
\hline IPF & $5(13 \%)$ & $4(40 \%)$ & $8(29 \%)$ & \\
\hline A1A deficiency & $5(13 \%)$ & & $1(3 \%)$ & \\
\hline Scleroderma/crest & $3(8 \%)$ & $1(10 \%)$ & $2(7 \%)$ & \\
\hline Sarcoidosis & $2(5 \%)$ & & & \\
\hline PPH & $1(3 \%)$ & & $1(3 \%)$ & \\
\hline Pulmonary fibrosis & $1(3 \%)$ & & & \\
\hline$E G$ & $1(3 \%)$ & & & \\
\hline OB/Retx & & $3(30 \%)$ & $1(3 \%)$ & \\
\hline Other & & & $2(7 \%)$ & \\
\hline Type of transplantation & & & & NS \\
\hline Single & $17(45 \%)$ & $5(50 \%)$ & $12(43 \%)$ & \\
\hline Double & $20(53 \%)$ & $4(40 \%)$ & $15(54 \%)$ & \\
\hline Heart, double lung & & $1(10 \%)$ & & \\
\hline Double lung + liver & $1(2 \%)$ & & & \\
\hline Living lung donor lobar & & & & \\
\hline CMV $(D+/ R-)$ & $13 / 38(34 \%)$ & $2 / 10(20 \%)$ & $7 / 28(25 \%)$ & NS \\
\hline $\mathrm{EBV}(\mathrm{D}+/ \mathrm{R}-)$ & $2 / 38(5 \%)$ & $0 / 10$ & $1 / 28(3 \%)$ & NS \\
\hline HLA mismatch & $4.5 \pm 1.3$ & $5.2 \pm 0.8$ & $5.2 \pm 0.9$ & NS \\
\hline $\mathrm{PRA}>10 \%$ & $1 / 38(3 \%)$ & $1 / 10(10 \%)$ & $2 / 28(7 \%)$ & NS \\
\hline Ischemia time (min) & $345 \pm 101$ & $354 \pm 85$ & $329 \pm 82$ & NS \\
\hline Donor age (y) & $35 \pm 15(14-63)$ & $39 \pm 16(11-62)$ & $35 \pm 15$ & NS \\
\hline
\end{tabular}

$C O P D$, Chronic obstructive pulmonary disease; $C F$, cystic fibrosis; IPF, idiopathic pulmonary fibrosis; $A 1 A$ deficiency, $\alpha_{1}$-antitrypsin deficiency; $P P H$, primary pulmonary hypertension; $E G$, eosinophilic granuloma; $O B / R e t x$, obliterative bronchiolitis retransplantation; $C M V$, cytomegalovirus; $E B V$, Epstein-Barr virus; $P R A$, panel reactive antibody.

\section{Assays of Lymphoid Depletion}

Patients receiving Thymoglobulin or Campath pretreatment were assessed for leukocyte subsets by means of flow cytometry at baseline (immediately before transplantation) and monitored for recovery at posttransplant days 1, 7, 14, and 30 and months 3 and 6 .

\section{Monitoring of Allograft Function}

In all cohorts surveillance spirometry and bronchoscopy with bronchoalveolar lavage and transbronchial biopsy were performed at 2 and 8 weeks after transplantation and then every 2 to 3 months, as well as when clinically indicated.

\section{Infection Monitoring and Prophylaxis}

Infection prophylaxis against cytomegalovirus (CMV) in Thymoglobulin- and Campath-treated patients consisted of 450 to $900 \mathrm{mg} / \mathrm{d}$ valganciclovir (Valcyte; Roche Laboratories, Nutley, $\mathrm{NJ})$ for 6 months. For prophylaxis against fungus and yeast, 6 $\mathrm{mg} / \mathrm{kg}$ voriconazole (Vfend; Pfizer, New York, NY) was administered intravenously every 12 hours for 2 doses, followed by $200 \mathrm{mg}$ twice daily for 4 months. Pneumocystis carinii prophylaxis was with one trimethoprim/sulfamethoxazole (TMP/sulfa) single strength tablet 3 times per week.
Daclizumab-treated patients received valganciclovir and TMP/ sulfa as described but received 3 months of fluconazole (Diflucan, Pfizer) prophylaxis in lieu of voriconazole.

\section{Spaced Reduction of Tacrolimus}

In 17 Thymoglobulin-pretreated recipients who were 6 months or more after transplantation, an effort was made to reduce tacrolimus dosing to 4 times a week or 3 times per week. The decisions were made either as a search for the lowest level of treatment consistent with stable graft function in response to low-level nephrotoxicity or because of concern about smoldering infection or incipient posttransplant lymphoproliferative disease.

\section{Informed Consent and Data Compilation}

Because the efficacy and safety of the immunosuppressive strategy had been demonstrated with other kinds of organ transplantation, ${ }^{6,7}$ the treatment regimen was deemed with the agreement of the University of Pittsburgh Institutional Review Board to be within the boundaries of standard therapy. The protocol was reviewed and authorized by the Presbyterian University Hospital Innovative Practices Committee and by the Pharmacy and Thera- 

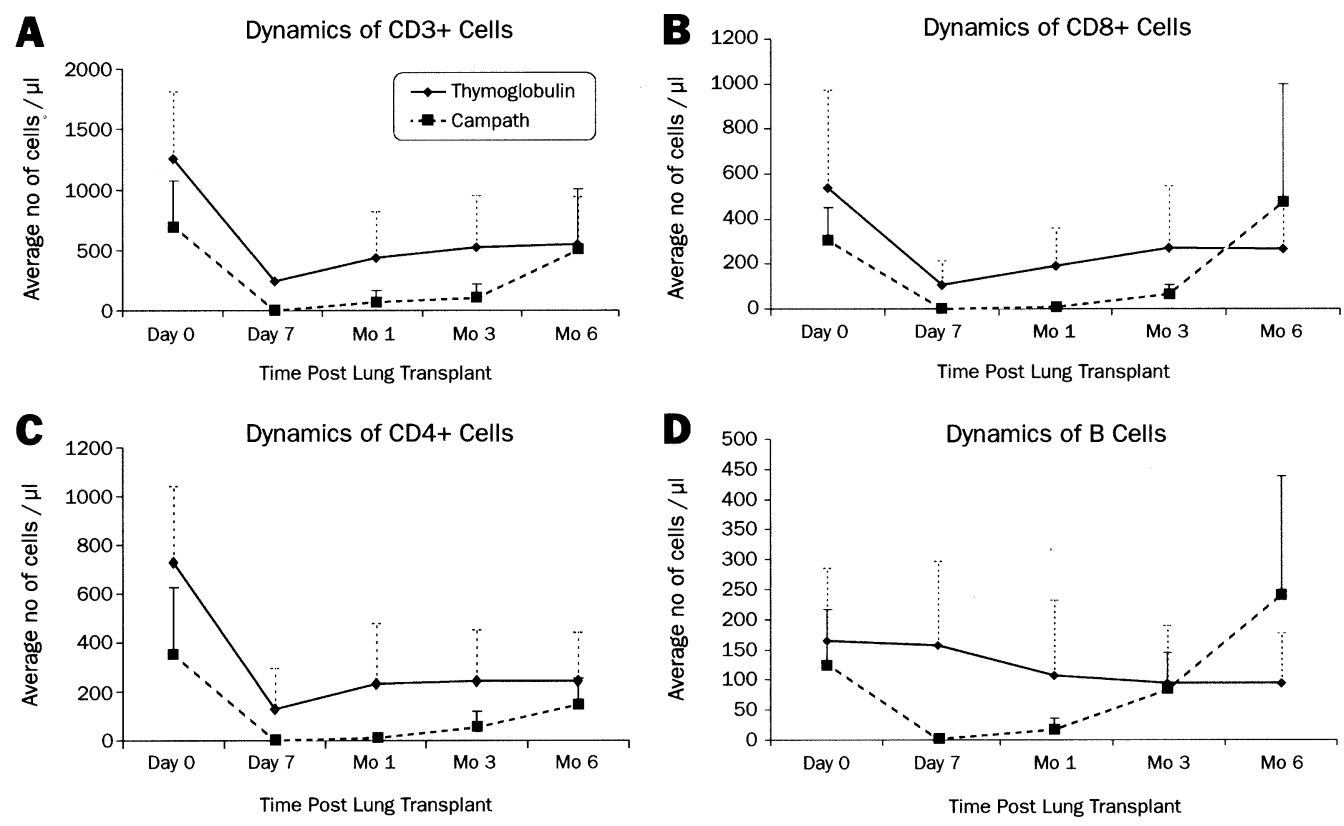

Figure 1. The effect of Thymoglobulin and Campath on leukocyte subsets and their recovery after transplantation. The dashed line represents the value of 100 cells $/ \mu \mathrm{L}$ (see text). ${ }^{16}$ Values are expressed as means \pm SD.

peutics Committee. Patients provided standard informed consent. In addition, all patients provided informed consent for enrollment under an institutional review board-approved protocol for studies not routinely performed in our conventional practice and for reporting of outcomes. Data were collected in a prospective manner. Data integrity and safety and efficacy monitoring were ensured by means of establishment of a formal weekly review of cases. Because Campath cases were compiled recently, the comparisons between the therapeutic antibodies are limited to 6 months.

\section{Statistical Analysis}

The $\chi^{2}$ statistic was used to determine the significance of nominal variables. Continuous variables were compared by either a Student $t$ test or a Kruskal-Wallis test. All $P$ values reported are 2 tailed. Survival and freedom-from-rejection analysis was determined by Kaplan-Meier actuarial analysis, and differences were compared with the log-rank test. Values are reported as means \pm SD.

\section{Results}

\section{Tolerability of Pretreatment Protocol}

The antibody infusions were begun as soon as possible after determination of the acceptability of donor organs. Frequently, this preceded initiation of general anesthesia. All infusions were completed before graft reperfusion. Thymoglobulin was infused over an average of $225 \pm 68$ minutes (range, 120-433 minutes), whereas Campath was infused over 2 hours. Both agents were generally well tolerated. In one patient with obstructive lung disease, significant bronchospasm began before starting Thymoglobulin and continued afterward. Thymo- globulin was transiently stopped and later resumed at a slower rate, allowing almost a full dose $(4 \mathrm{mg} / \mathrm{kg})$ to be administered before allograft reperfusion. Another patient who received Thymoglobulin had significant hyperthermia, resulting in slowing of the rate of infusion.

\section{Immune Cell Reconstitution}

Pretreatment with 4 to $7 \mathrm{mg} / \mathrm{kg}$ Thymoglobulin resulted in profound depletion of $\mathrm{T}$ cells $\left(\mathrm{CD}^{+}\right)$by postoperative day 1 . T-cell counts remained less than baseline values for 3 months, with a gradual return toward normal levels by 3 to 6 months after transplantation (Figure 1, $A$ ). The CD8 population recovered more rapidly than the CD4 population. Although CD8 cell counts returned to baseline by 1 to 3 months after transplantation, CD4 cell counts remained at less than baseline value for up to 6 months (Figure 1,B-C). This pattern resulted in an inverted $\mathrm{CD} 4 / \mathrm{CD} 8$ ratio that persisted up to 6 months after transplantation. B cells were not depleted by Thymoglobulin (Figure 1, D).

The level of T-cell depletion achieved was significantly greater and more sustained with Campath than with Thymoglobulin (Figure 1, A) and included CD8 and CD4 phenotypes (Figure $1, B-C$ ). CD4 (helper) cells were the most profoundly affected. The mean value for $\mathrm{CD}^{+}$cell counts depicted in Figure 1, A, at 6 months was due to high cell counts in 2 patients. The remainder of patients had cell counts that were 290 cells/ $\mu \mathrm{L}$ or less. 
- Complete + Censored

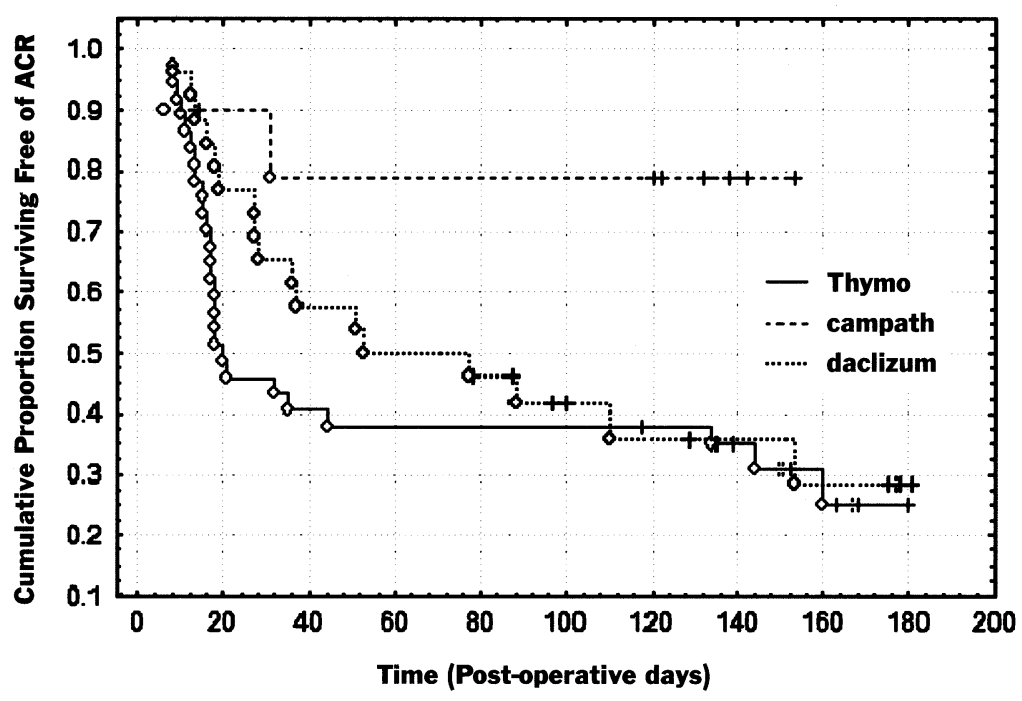

Figure 2. Kaplan-Meier plot of freedom from grade 2 or greater rejection. ACR, Acute cellular rejection.

Unlike Thymoglobulin, B cells were significantly depleted with Campath but returned to normal levels by 3 months (Figure 1,D).

\section{Rejection Episodes}

The incidence and overall burden of acute rejection was compared in patients who survived at least 2 weeks after transplantation (37/38 Thymoglobulin-treated patients, 10/10 Campath-treated patients, and 26/28 daclizumabtreated patients). The percentage of patients with at least one episode of grade 2 or greater acute rejection in the first 6 posttransplant months was no different between the Thymoglobulin- and daclizumab-treated cohorts $(25 / 37$ [68\%] vs $17 / 26$ [65\%], $P=$ not significant [NS]). In contrast, the Campath cohort had significantly fewer patients with rejection $(2 / 10$ [20\%]; $P=.008$ vs Thymoglobulin and $P=.02$ vs daclizumab). Similarly, there was no difference in the percentage of patients with high-grade rejection ( $\geq$ grade 3 ) between the Thymoglobulin- and daclizumab-treated cohorts (16/37 [43\%] vs $12 / 26$ [46\%], $P=$ NS), whereas no Campath-conditioned patients had high-grade rejection $(P=.01 \mathrm{vs}$ Thymoglobulin and $P=.01$ vs daclizumab). Figure 2 shows the Kaplan-Meier plot of freedom from greater than or equal to grade 2 rejection, demonstrating the lower incidence of rejection in the Campath cohort.

In addition, in those patients who experienced rejection, the number of rejection episodes was fewer in the Campath cohort compared with the Thymoglobulin and daclizumab cohorts. The average number of grade 2 or greater episodes of rejection per patient was 1.45 in the Thymoglobulin cohort and 1.15 in the daclizumab cohort $(P=$ NS) but was significantly less in Campath-treated patients $(0.2$ episodes per patient [ie, a single episode of grade 2 rejection in 2 patients], $P=.007$ vs Thymoglobulin and $P=.03$ vs daclizumab). Neither Campathpreconditioned patient was treated with augmented immunosuppression for the grade 2 rejection. The frequency of high-grade rejection episodes was similarly no different between Thymoglobulin- and daclizumab-treated patients ( 0.81 vs 0.57 episodes per patient, respectively; $P$ $=$ NS) but was significantly less in Campath-treated patients ( 0 episodes, $P=.02$ vs Thymoglobulin and $P=$ .032 vs daclizumab). No patients in any group had histopathologic evidence of obliterative bronchiolitis during the first 6 months.

\section{Survival}

Six-month survival was not statistically different between the groups. Although survival in the Thymoglobulin and Campath groups was greater than our previous 3-year experience, the difference did not reach statistical significance (Figure 3). Six-month survival in the Thymoglobulin-treated cohort was 97\% (37/38). The only death during this period occurred on postoperative day 9 because of the sequelae of ischemiareperfusion injury. One patient required early retransplantation (postoperative day 54) for allograft dysfunction after transplantation for sarcoidosis. The patient survived. Thus, graft survival at 6 months was $95 \%(36 / 38)$. 


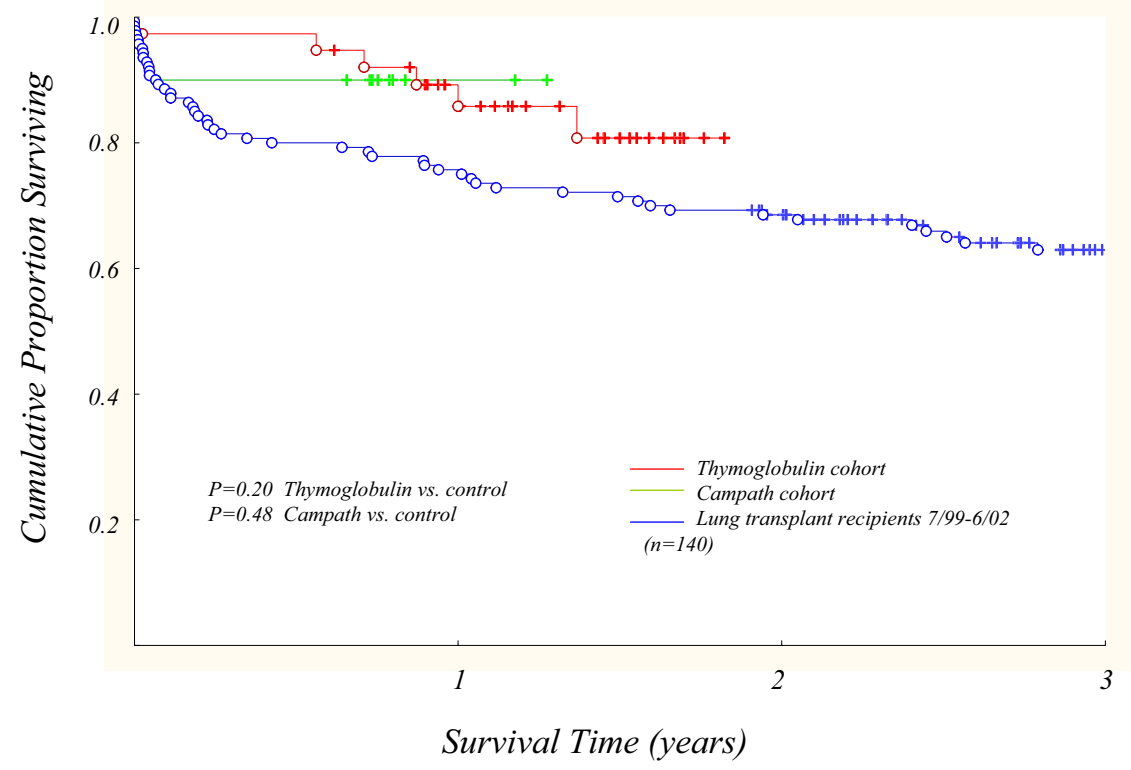

Figure 3. Kaplein-Meier actuarial survival curves for the Thymoglobulin and Campath cohorts, as well as for the group of patients receiving lung transplants at the University of Pittsburgh Medical Center from July 1999 to June 2002. Although pretreatment with either Thymoglobulin or Campath with resultant management, as described in the text, resulted in a greater than $10 \%$ difference in 1-year survival between the Thymoglobulin and Campath groups and the historical group, the difference did not reach statistical significance. This is likely because of the small sample size.

Six-month survival in the Campath-treated cohort was 90\% (9/10). The only death occurred on postoperative day 23 in a 70-year-old recipient of a double-lung transplant (for emphysema) after an apneic episode on the ward.

Six-month survival in the daclizumab group was $89 \%$ (25/28).

\section{Pulmonary Function}

The average forced expiratory volume in 1 second $\left(\mathrm{FEV}_{1}\right)$ in the Thymoglobulin-treated cohort at 6 months was $70 \%$ $\pm 19 \%$, whereas that in the Campath-treated cohort was $80 \% \pm 27 \%(P=\mathrm{NS})$. One single-lung recipient in the Thymoglobulin-pretreated group reached a peak $\mathrm{FEV}_{1}$ at 2 months after transplantation, with a subsequent decrease in values. Although transbronchial and open lung biopsies have not revealed any evidence of pathologic obliterative bronchiolitis, the $\mathrm{FEV}_{1}$ values meet bronchiolitis obliterans syndrome 2 criteria. ${ }^{8}$ This patient had preexisting donorspecific anti-HLA class II antibody (Table 1). No Campathtreated patients met bronchiolitis obliterans syndrome criteria.

\section{Infection}

There was no significant difference in infection rates among the 3 groups, although there was a trend toward less CMV- induced disease in the Campath cohort. Eighteen percent $(5 / 28)$ of patients in the daclizumab group, compared with $8 \%(3 / 38)$ in the Thymoglobulin group and $0 \%$ in the Campath group, had histologically proved CMV-induced disease $(P=.23)$. There were 3 cases of Nocardia species (one each of Nocardia Nova, Nocardia farcinica, and Nocardia asteroides complex) recorded in patients receiving Thymoglobulin pretreatment, whereas none occurred in the other groups $(P=.19)$.

\section{Survival After 6 Months}

The potential follow-up for the 38 Thymoglobulinpretreated recipients is now 9 to 23 months. Although 37 patients were alive at 6 months, 3 more deaths occurred by 1 year ( 2 caused by sepsis and 1 caused by posttransplant lymphoproliferative disease), and 2 additional deaths occurred after 13 and 16 months (viral influenza and chronic rejection, respectively). Thus, the 1-year actuarial survival is $86 \%$. Thirty-two $(84.2 \%)$ of the original 38 patients are alive, with a mean follow-up of $17.1 \pm 4.0$ months. Except for the death at 3 weeks of a 70-year-old emphysematous recipient, all of the other Campath-pretreated patients are alive, with a mean follow-up of $10.5 \pm 4.4$ months (range, 9-17 months). 


\section{Spaced Reduction of Tacrolimus}

In 16 of the 32 surviving patients pretreated with Thymoglobulin, an attempt was made to reduce tacrolimus doses to 3 or 4 times a week. Five of the 16 patients remain stable on spaced dosing after a mean period of $8.5 \pm 2.8$ months (range, 6-12 months). Weaning was discontinued in the other 11 patients because of decreases in pulmonary function or because of histopathologic evidence of destructive immunity on biopsy. Despite augmentation of immunosuppression, full restoration of baseline function was achieved in only 3 of these patients. In contrast, the other 16 surviving recipients of the Thymoglobulin cohort who have been maintained on daily (twice a day or once a day dosing) tacrolimus monotherapy or near-monotherapy have had no deterioration in pulmonary function after a mean follow-up of $14.6 \pm 3.7$ months.

\section{Discussion}

There are obvious limitations to this study. Because it was a phase I-II study rather than a prospective randomized trial, definitive comparisons could not be made with contemporaneous (or, in our report, historical) control patients treated with conventional multiple drug immunosuppression. In addition, follow-ups are still short, particularly for the cohort of Campath-pretreated recipients. Nevertheless, it is noteworthy that our 6-month patient survival (97\% and 90\% in the Thymoglobulin and Campath cohorts, respectively) is competitive with the 89\% 6-month survival with triple-drug immunosuppression in our immediately preceding experience and the benchmark $79 \%$ of the ISHLT registry. ${ }^{2}$ Moreover, current survival is $84.2 \%$ of our Thymoglobulinpretreated recipients with follow-ups of 9 to 23 months and has remained at $90 \%$ since postoperative week 3 in the Campath-pretreated patients who are now 9 to 17 months after transplantation. In addition, no further deaths have occurred as additional patients $(>40)$ have received transplants with Campath preconditioning. These results were achieved without an increased risk of infection and, in fact, with a trend toward less CMV-induced disease in an unselected series of patients who had a large representation of high-risk factors.

Both Thymoglobulin and Campath are potent lymphoiddepleting agents. Thymoglobulin ${ }^{9,10}$ has properties similar to the original polyclonal antilymphoid globulins that were introduced clinically nearly 40 years ago, and the more T-cell specific muromonab (OKT3). Campath was developed by Hale and Waldmann ${ }^{11}$ and first used for kidney transplantation by Calne and colleagues. ${ }^{12}$ In accord with reports by Knechtle, ${ }^{13}$ Kirk, ${ }^{14}$ and their associates, our data demonstrate that Campath has a more profound and prolonged lymphoid depletion than Thymoglobulin, including depression of the B-lymphocyte and natural killer lineages without causing major decreases in platelet counts. The
Campath effect lasted for 6 months compared with the few weeks of Thymoglobulin. Because the extent and duration of T-cell depletion is thought to correlate with the avoidance of rejection, ${ }^{15}$ including that of thoracic organs, ${ }^{16}$ the lower rate of rejection in the Campath cohort relative to the antilymphoid globulin group was not surprising.

Moreover, the acute rejections were less severe in the patients pretreated with Campath. The incidence of grade 2 or higher acute rejection in the Thymoglobulin-treated patients was $66 \%$, although the treated acute rejection rate was $47 \%$. The incidence of rejection was similar to that seen with standard triple immunosuppression without use of antibody induction, ${ }^{17}$ as well as in some series in which antilymphoid globulins were administered postoperatively. ${ }^{1}$ In contrast, only 2 of the 10 Campath-pretreated patients had 1 episode each of grade 2 acute rejection (neither treated), and no patients had grade 3 rejection or higher. These patients were treated with steroid boluses or a short course of another agent but otherwise managed throughout with low maintenance immunosuppression. Five of the 10 recipients treated with Campath never were administered anything but tacrolimus, whereas the other 5 also were given $5 \mathrm{mg} / \mathrm{d}$ or less prednisone, usually because the patients previously had received chronic steroid therapy.

The timing of the lymphoid depletion in our patient deserves particular emphasis. The theory underlying our treatment strategy is that the seminal mechanism of organ alloengraftment is passenger leukocyte-driven clonal exhaustion-deletion, ${ }^{18}$ an immune activation-dependent mechanism that can be variably eroded by heavy prophylactic postoperative immunosuppression. ${ }^{4}$ Lymphoid depletion before exposure to donor alloantigen was designed to avoid this pitfall, reduce the anticipated donor-specific response into a more deletable range, and allow the safe use of minimum posttransplant immunosuppression. Thus our hypothesis was (and is) that organ engraftment is a state of variable partial tolerance that can be made more complete by modifying the timing and dosage of immunosuppression.

Our view of organ engraftment and its relation to acquired tolerance has been controversial. A large body of historical and recent work has led to alternative hypotheses to explain organ engraftment, acquired tolerance, or both. ${ }^{19,20}$ These theories emphasize the importance of immunoregulatory $\mathrm{T}$ cells, other changes in the host immune response, or both that can downregulate alloimmune or autoimmune responses. In addition to T-cell immunoregulation, subsets of other special cells, changes in the host cytokine profile, or the development of enhancing antibodies might play a role. In an attempt to foster these mechanisms, lymphoid depletion has been done elsewhere at and after the time of transplantation. In recent trials Campath (or Thymoglobulin) was administered in 2 doses: the first intraoperatively and the second a few days later. Preliminary 
results, especially after renal transplantation, have been encouraging. ${ }^{12-14}$

Our strategy of lymphoid depletion before, rather than at or after, transplantation also has been shown to be effective for kidney, liver, and other kinds of organ transplantation. ${ }^{6,7,21}$ Implementing this regimen in a clinical setting of thoracic organ transplantation entailed 2 concerns. The first question was whether the use of minimalist posttransplant immunosuppression might result in an unacceptable loss of allografts to uncontrollable acute rejection. In the experience reported herein, the risk of short-term graft loss to acute rejection was no greater than with conventional immunosuppression in either the Thymoglobulin- or Campathpretreated recipients.

The second question concerned the extent to which daily minimal maintenance immunosuppression could be sustained after recovery from the lymphoid depletion or reduced further, without the penalty of breakthrough acute rejection or indolent chronic rejection. Studies in mice by $\mathrm{Wu}$ and colleagues ${ }^{22}$ have raised the possibility that recovery from lymphoid depletion (homeostatic proliferation) might be associated with heightened long-term immune reactivity. The adverse consequences could include vigorous later rejection that could become evident only with long follow-up. This issue can be tentatively addressed with observations in the Thymoglobulin-pretreated recipients, 32 of whom survive after 17.1 \pm 4.0 months (range, 9-23 months).

Fifteen of the 32 patients have been maintained on tacrolimus monotherapy for $14.6 \pm 3.7$ months (range, 9-22 months), with no deterioration of graft function. An additional patient who had been maintained on twice-daily tacrolimus had a late rejection episode (11 months) and required the addition of mycophenolate mofetil after treatment with steroids. In 16 others in whom spaced reduction of tacrolimus was attempted, this was tolerated by only 5 patients. Therefore we have concluded that attempts at spaced reduction of tacrolimus should not be made unless there is a specific reason to do so (eg, calcineurin inhibitorrelated renal dysfunction or an ongoing infection). A similar approach is being taken in the Campath-pretreated patients. We emphasize, however, that much longer follow-ups will be needed before the threat posed by chronic rejection can be fully evaluated.

Randomized trials comparing the results with the different timing of antilymphoid antibody treatment, as well as comparisons of the lymphoid-depleting strategies with conventional immunosuppression, could lead to better care of lung recipients. In addition to analyses of clinical outcomes, detailed immune monitoring in such trials might help resolve disputes about the mechanisms of engraftment. Finally, the observations with lung transplantation are expected to be generalizable to other or- gans, including the heart. We have pretreated 3 heartlung recipients with Campath (one included in the current report) and a recent heart-only recipient. All 4 patients have been free of rejection on tacrolimus monotherapy.

We thank Terry L. Mangan for secretarial assistance and all members of the University of Pittsburgh Lung and Heart-Lung Transplantation Program for their dedication to patient care.

\section{References}

1. Brock MV, Borja MC, Ferber L, Orens JB, Anzcek RA, Krishnan $\mathrm{J}$, et al. Induction therapy in lung transplantation: a prospective controlled trial comparing OKT3, anti-thymocyte globulin and daclizumab. J Heart Lung Transplant. 2001;20:1282-90.

2. Trulock EP, Edwards LB, Taylor DO, Boucek MM, Mohacsi PJ, Keck $\mathrm{BM}$, et al. The registry of the International Society for Heart and Lung Transplantation: Twentieth official adult lung and heart-lung transplant report-2003. J Heart Lung Transplant. 2003;22:625-35.

3. The U.S. Organ Procurement and Transplantation Network and The Scientific Registry of Transplant Recipients: transplant data 19922001. Washington (DC): Department of Health and Human Services; 2002 Annual Report.

4. Starzl TE, Zinkernagel RM. Transplantation tolerance from a historical perspective. Nat Rev Immunol. 2001;1:233-9.

5. Yousem SA, Berry GJ, Cagle PT, Chamberlain D, Husain AN, Hruban $\mathrm{RH}$, et al. Revision of the 1990 working formulation for the classification of pulmonary allograft rejection: Lung Rejection Study Group. J Heart Lung Transplant. 1996;15:1-15.

6. Starzl TE, Murase N, Abu-Elmagd K, Gray EA, Shapiro R, Eghtesad $\mathrm{B}$, et al. Tolerogenic immunosuppression for organ transplantation. Lancet. 2003;361:1502-10.

7. Shapiro R, Jordan ML, Basu A, Scantlebury V, Potdar S, Tan HP, et al. Kidney transplantation under a tolerogenic regimen of recipient pretreatment and low-dose postoperative immunosuppression with subsequent weaning. Ann Surg. 2003;238:520-7.

8. Cooper JD, Billingham M, Egan T, Hertz MI, Higgenbottam T, Lynch $\mathrm{J}$, et al. A working formulation for the standardization of nomenclature and for clinical staging of chronic dysfunction in lung allografts. International Society for Heart and Lung Transplantation. J Heart Lung Transplant. 1993;12:713-6.

9. Preville X, Flacher M, LeMauff B, Beauchard S, Davelu P, Tiollier J, et al. Mechanisms involved in antithymocyte globulin immunosuppressive activity in a nonhuman primate model. Transplantation. 2001;71:460-8.

10. Mueller TF. Thymoglobulin: an immunologic overview. Curr Opin Organ Transplant. 2003;8:305-12.

11. Hale G, Waldmann H, Dyer M. Specificity of monoclonal antibody Campath-1H. Bone Marrow Transplant. 1988;3:237-9.

12. Calne R, Friend P, Moffatt S, Bradley A, Hale G, Firth J, et al. Prope tolerance, perioperative Campath $1 \mathrm{H}$, and low-dose cyclosporin monotherapy in renal allograft recipients [published erratum appears in Lancet. 1998;352:408]. Lancet. 1998;351:1701-2.

13. Knechtle SJ, Pirsch JD, Fechner HJ Jr, Becker BN, Friedl A, Colvin $\mathrm{RB}$, et al. Campath-1H induction plus rapamycin monotherapy for renal transplantation: results of a pilot study. Am J Transplant. 2003; 3:722-30.

14. Kirk AD, Hale DA, Mannon RB, Kleiner DE, Hoffmann SC, Kampen $\mathrm{RL}$, et al. Results from a human renal allograft tolerance trial evaluating the humanized CD52-specific monoclonal antibody alemtuzumab (CAMPATH-1H). Transplantation. 2003;76:120-9.

15. Cosimi AB, Wortis HH, Delmonico FL, Russell PS. Randomized clinical trial of antithymocyte globulin in cadaver renal allograft recipients: importance of T cell monitoring. Surgery. 1976;80:155-63.

16. Krasinskas AM, Kreisel D, Acker MA, Bavaria JE, Pochettino A, Kotloff RM, et al. CD3 monitoring of antithymocyte globulin therapy in thoracic organ transplantation. Transplantation. 2002;73:1339-41.

17. Bhorade SM, Jordan A, Villanueva J, Yu A, Kramer H, Vigneswaran WT, et al. Comparison of three tacrolimus-based immunosuppressive regimens in lung transplantation. Am J Transplant. 2003;3:1570-5. 
18. Starzl TE, Zinkernagel RM. Antigen localization and migration in immunity and tolerance. $N$ Engl J Med. 1998;339:1905-13.

19. Walker LSK, Abbas AK. The enemy within: keeping self-reactive $T$ cells at bay in the periphery. Nat Rev Immunol. 2002;2:11-9.

20. Lechler RI, Garden OA, Turka LA. The complementary roles of deletion and regulation in transplantation tolerance. Nat Rev Immunol. 2003;3:147-58.

21. Marcos A, Eghtesad B, Fung JJ, Fontes P, Patel K, deVera M, et al. The use of alemtuzumab and tacrolimus monotherapy for cadaveric liver transplantation. Transplantation. 2004;78:966-71.

22. Wu Z, Bensinger SJ, Zhang J, Chen C, Yuan X, Huang X, et al. Homeostatic proliferation is a barrier to transplantation tolerance. Nat Med. 2004;10:87-92.

\section{Discussion}

Professor Bruce Rosengard (Cambridge, United Kingdom). I would like to first congratulate Dr McCurry and his colleagues at Pittsburgh for the courage to apply the pioneering work of my colleague, Professor Sir Roy Calne, to lung transplantation. As we all know, many of the early obstacles to lung transplantation have been overcome, but as you so rightly pointed out, the long-term outcomes caused by obliterative bronchiolitis remain a plague of the lung transplant recipient. To the extent that obliterative bronchiolitis is caused by an immune response, there is no patient group in transplantation that is more in need of better immunosuppressive therapy.

Sir Roy's concept of Prope or almost tolerance has now been applied to several hundred liver or kidney recipients worldwide and is presently undergoing phase III testing to establish efficacy in comparison with conventional triple-drug immunosuppression. Indeed, of Sir Roy's original 29 kidney patients treated with Campath and cyclosporine monotherapy, 26 remain on low-dose cyclosporine monotherapy, and the other 3 have had azathioprine and steroids added back. All patients at more than 5 years' follow-up now are doing very well clinically, with virtually normal renal function.

If the majority of lung recipients are able to be treated with long-term monotherapy after an intense lymphocyte cytoreduction, this will be a major clinical advance because side effects and long-term complications will be reduced, even though the approach does not achieve true tolerance. I have 3 questions for the authors.

In your article you attribute your clinical observations to clonal deletion or exhaustion of alloreactive T-cell clones; however, other clinical investigators have shown persistence of alloreactive clones after Campath induction followed by tacrolimus monotherapy. Do you have evidence for either clonal deletion or exhaustion, or is it more likely that your protocol allows for the emergence of regulatory $\mathrm{T}$ cells, which mute the immune response?

Dr McCurry. Thank you very much, Bruce, for your kind comments, and I would also like to take the opportunity from a personal standpoint to thank you for your contributions and leadership in thoracic transplantation and personally for some support that you provided to me.

Your question is a very good one. Our paradigm has been that of clonal exhaustion deletion, meaning that passenger leukocytes migrate from the organ and go through a process with diminished initial alloimmune responses of clonal proliferation and ultimate exhaustion. This is based on historical work at our institution, as well as some recent work that has been done in kidneys and livers. We have little data in the lung transplant population to demonstrate or suggest that this is definitely the mechanism by which this is occurring.

We have done some early in vitro responses, and we do have 2 patients who have diminished, not absent, responses, but who are hyporesponsive by mixed leukocyte reaction in vitro; however, there are certainly many other patients who maintain in vitro alloreactivity. In addition, we do have 2 patients who show a diminished frequency of donor-specific reactive $\mathrm{T}$ cells, as determined by means of limiting dilution assay, but again, many do not. Therefore, I think what we see is that for some reason, many of these patients are able to be maintained on low-dose monotherapy.

The issue of whether we are inducing a regulatory $\mathrm{T}$ cell is a very good question also. As I am sure you well know, Stewart Knechtle and Alan Kirk have used Campath induction in kidney recipients, and I believe that both groups have recently reported the demonstration or a suggestion of development of a regulatory $\mathrm{T}$ cell in patients who have been treated with Campath who are now more than a year out from their transplantations.

Most of our Campath-treated patients are relatively early after transplantation at this point, but this is certainly an area that we are interested in and an area that we are beginning to look at, looking for both the $\mathrm{CD} 4^{+}, \mathrm{CD} 25^{+}$regulatory cell and the $\mathrm{CD} 8^{+}$population that the Columbia group has identified. In addition, we have also been looking for FOXP3, as well, which, as you know, is the marker of perhaps a tolerant phenotype.

Professor Rosengard. Second, there is emerging evidence that $\mathrm{T}$ cells that repopulate a recipient after intense lymphocyte cytoreduction acquire the phenotype of memory or immunized cells, even in the absence of alloantigen. Do you think that this will limit the long-term efficacy of this approach because memory $\mathrm{T}$ cells are notoriously resistant to immunosuppressive therapy?

Dr McCurry. Another very good question and something that we are concerned about. I think most of this work demonstrating repopulation with a memory phenotype, as has been done by yourself, as well as Larry Turk and the group at Emory, which we have certainly read with interest, is an area that we are just now beginning to look at in our patients. We really do not have any data, but we are trying to look at the phenotype of the cells that do repopulate after both Thymoglobulin and Campath treatment. I do not know. It is hard to say. Certainly a mouse is not a man, and I think that Sir Roy Calne's data perhaps would argue that it is not a concern in human transplantation, although certainly a kidney is not a lung either.

Professor Rosengard. Finally, I am happy to see that you are planning to do a prospective randomized study. I was going to ask you, given the fact that hundreds of patients have been studied with phase I protocols in kidney and liver transplantation, why was it that you chose to do a phase I study in lung recipients as opposed to going for the full Monty?

Dr McCurry. I think for several reasons. One is, obviously, as this group well knows, that lungs are difficult, and the early outcomes after lung transplantation have been notoriously difficult. We thought it best to obtain some safety data as an initial foray into this at a single center. I think it is also true that in 
general the thoracic transplant community has not believed either from an immunologic or a safety standpoint a lot of the work that has been done in abdominal transplantation. A recent application of this, that is, the use of rapamycin after trials in kidneys, resulted in some bad outcomes in lung transplant recipients with bronchial dehiscence. Therefore we believed it was best to pursue a safety trial initially at our center, and then, on the basis of these data, we hope to proceed to a multicenter randomized trial.

Dr Thomas M. Egan (Chapel Hill, NC). Dr McCurry, congratulations on some outstanding results. My question does not relate to the immunologic aspect so much as a dramatic lack of problems with reperfusion injury. Most early mortality in lung transplantation relates to graft failure, and at least in your Campath group, it appears that you have avoided that. Is that just blind luck, or are you actually seeing a reduction in reperfusion injury as evidenced by ventilation duration, et cetera?

Dr McCurry. Thanks very much, Tom. It is an area that we also have been interested in, and I am sure part of it is blind luck, but there are some data, as I am sure you are well aware, that at least Thymoglobulin, the polyclonal agents, decrease ischemia-reperfusion injury in kidney transplantation, and our anecdotal results thus far with Campath have been that the early graft function is quite good. It is something that we are interested in at least from a clinical standpoint, comparing with some retrospective cohorts.

Dr Egan. Does that imply that the $\mathrm{T}$ cell is involved in that reperfusion injury?

Dr McCurry. There are certainly experimental data in animals to suggest that, and it might very well be.

Dr Alec Patterson (St Louis, Mo). Ken, that was an excellent presentation, very nicely delivered. If I understood or interpreted your slides correctly, the 1-year early survival really was not much different than what is reported in most experienced centers. Therefore although this strategy might have some relevance to bronchiolitis obliterans syndrome in later follow-up, I would have thought that we would see a reduction in early postoperative complications. Can you comment on that?

Dr McCurry. Thank you, Alec, and thank you and your group for your contributions and your leadership. We only have Thymoglobulin-treated patients who are 1 year out, and our 1-year survival in that patient population is $87 \%$, so it compares favorably with ISHLT data. But as you suggest, many experienced centers report 1-year survival of greater than $80 \%$.

Certainly during the early course of using Thymoglobulin in this patient population, we went through a learning curve, and although we had one death in that group in the first 6 monthstherefore we had 37 of 38 survivors in the Thymoglobulin group at 6 months-we did have another 3 deaths in the subsequent 6 months, and if I recall correctly, I believe that 2 of those deaths were due to sepsis and a third was due to a viral-related process, adult respiratory distress syndrome. It was a learning curve through that process.

I believe our results right now with Campath have been quite good, and we do have about 37 patients who are doing well, and I anticipate we will have a very good 1-year survival in that patient population. Again, only time will tell.

Dr Duane R. Davis, Jr (Durham, NC). I rise to congratulate you for having the courage to try to change the paradigm of immunosuppression. Dr Alan Kirk, in his experience with Campath in kidney transplant recipients, noted an acute rejection process that was dominated histologically by macrophage and monocytes. You have demonstrated that your patients treated with Campath have no $\mathrm{T}$ cells. What are the cells that are infiltrating the graft? As a secondary comment, what is the difference between peripheral and central depletion between Thymoglobulin and Campath?

Peripheral depletion tends to be pretty good with both. However, central compartment depletion, which is probably going to be much more important in terms of being able to approach a tolerogenic or at least a minimal immunosuppression strategy, probably is significantly better with Campath than with Thymoglobulin.

Dr McCurry. Thank you, Duane. Those are both very interesting questions. I think, as you suggest, when Alan published his data, of course he used Campath induction, I believe, if I recall correctly, 2 doses, about $40 \mathrm{mg}, 50 \mathrm{mg}$, perhaps, with no baseline immunosuppression afterward. What we have typically seen in the Campath-treated patients is a bit of an early alloimmune response that has been a typical mononuclear $\mathrm{T}$ cell infiltrate.

Now we have not done the immunostains and the typing with immunohistochemistry that Alan did in his study, but certainly the appearance of the grade 2 cases that we have seen look like other grade 2 cases that we have seen with Campath. Now that might be because perhaps we are not depleting as much as Alan was because we are giving a smaller dose, a single 30-mg dose as opposed to multiple doses up to 40 or $50 \mathrm{mg}$, but we really have not appreciated a difference from a histologic standpoint.

Your second question about central depletion is a very good question. Our paradigm has been to try to get all of the Campath, or whatever agent we are using, in before allograft reperfusion. Again, we are using a smaller dose of Campath than most of the abdominal groups have; I believe Alan has used up to $60 \mathrm{mg}$ in his patient population, and Stewart perhaps used a similar dosing. Therefore, ideally we are depleting centrally. We do not have data to demonstrate that, but certainly from a peripheral standpoint, it takes a long time to reconstitute the CD8 population, particularly the CD4 population. 